\title{
Myocarditis associated with 2009 influenza A (H1N1) virus in children
}

Received: 8 December 2009; Accepted: 2 January 2010; First published online: 15 April 2010

Sir,

The very interesting articles reporting characteristics of hospitalised patients with 2009 influenza A (H1N1) encouraged us to write this commentary letter. In the main cohorts of 2009, H1N1 adult and paediatric patients published to date, no viral myocarditis has been reported. ${ }^{1-9}$ However, a large number of patients required intensive care unit admission and mechanical ventilation for acute respiratory distress. Most of them presented with arterial hypotension or shock requiring inotropic support or extracorporeal membrane oxygenation. ${ }^{3}$

We would like to share our experience in our paediatric intensive care unit, where three hospitalised children developed myocarditis during the epidemic wave in Reunion Island (Indian Ocean) from July, 2009 to October, 2009, epidemic peak week 30 to 38. Myocarditis was fulminant in two cases, presenting with an acute-onset heart failure and cardiogenic shock. Clinical characteristics of these three patients are summarised in Table 1. Influenza A (H1N1) was confirmed by specific reverse transcription - polymerase chain reaction on naso-pharyngeal swabs and by serologic analysis.

Data on the prevalence of myocarditis in children are limited and autopsy studies have shown that it often stayed undiagnosed. Myocarditis may be seen in severe forms of many infectious diseases.

Our report demonstrates that the 2009 Influenza A $(\mathrm{H} 1 \mathrm{~N} 1)$ virus is cardiotropic and emphasises that a prompt cardiologic examination including a transthoracic echocardiography is warranted in H1N1-infected patients in case of acute respiratory distress or haemodynamic instability. If fulminant myocarditis is recognised and patients aggressively supported in a timely manner, full recovery can be obtained with a low

Table 1. Clinical characteristics of $2009 \mathrm{H} 1 \mathrm{~N} 1$ myocarditis in paediatric patients, Reunion Island.

\begin{tabular}{|c|c|c|c|}
\hline & Patient 1 & Patient 2 & Patient 3 \\
\hline Patients & 9 years (male) & 12 years (female) & 7 months (female) \\
\hline Comorbidity & $\begin{array}{l}\text { Congenital subvalvular } \\
\text { mild aortic stenosis }\end{array}$ & $\begin{array}{l}\text { Severe cerebral palsy with } \\
\text { recurrent pulmonary infections }\end{array}$ & None \\
\hline Myocarditis presentation & $\begin{array}{l}\text { Acute-onset heart } \\
\text { failure and cardiogenic } \\
\text { shock }\end{array}$ & $\begin{array}{l}\text { Acute-onset heart failure and } \\
\text { cardiogenic shock }\end{array}$ & $\begin{array}{l}\text { Dilated cardiomyopathy without } \\
\text { hemodynamic instability }\end{array}$ \\
\hline Elevation of cardiac enzymes & Yes & Yes & Yes \\
\hline LVEF & $15 \%$ & $35 \%$ & $15 \%$ \\
\hline $\begin{array}{l}\text { Duration of inotropic support/ } \\
\text { ventilatory support (days) }\end{array}$ & $15 / 10$ & $5 / 30$ & $5 / 0$ \\
\hline $\begin{array}{l}\text { During of antiviral therapy/ } \\
\text { hospitalisation in PICU } \\
\text { (days) }\end{array}$ & $10 / 17$ & $15 / 30$ & $5 / 7$ \\
\hline Outcome & Full recovery & Full recovery & $\begin{array}{l}\text { Severe persistent depressed LVEF } \\
(25 \%) \text { after } 2 \text { months }\end{array}$ \\
\hline
\end{tabular}

$\mathrm{LVEF}=$ left ventricular ejection fraction, PICU $=$ paediatric intensive care unit

Correspondence to: Dr A.-E. Baruteau, MD, Service de Réanimation Néonatale et Pédiatrique, Centre Hospitalier Régional Félix Guyon, Allée des Topazes, 97405 SaintDenis de La Réunion, France. Tel: 02629058 30; Fax: 02629058 32; E-mail: alban.baruteau@wanadoo.fr 
mortality rate. ${ }^{10}$ We hope that sharing information in this field would be useful for the management of critically-ill H1N1-infected patients.

$$
\begin{array}{r}
\text { Alban-Elouen Baruteau, }{ }^{1,2} \text { Nicolas Boimond, }{ }^{1} \\
\text { Duksha Ramful }
\end{array}
$$

${ }^{1}$ CHR Felix Guyon, Service de Réanimation Néonatale et Pédiatrique, Saint-Denis de La Réunion F-97405, France

${ }^{2}$ Centre Chirurgical Marie Lannelongue, Service des Cardiopatbies Congénitales, Le Plessis Robinson F-92350, France

\section{References}

1. Dominguez-Cherit G, Lapinsky SE, Macias AE, et al. Criticallyill patients with 2009 Influenza A (H1N1) in Mexico. JAMA 2009; 302: 1880-1887.

2. Kumar A, Zarychanski R, Pinto R, et al. Critically-ill patients with 2009 Influenza A (H1N1) in Canada. JAMA 2009; 302: 1872-1879.
3. Lister P, Reynolds F, Parslow R, et al. Swine-origin influenza virus $\mathrm{H} 1 \mathrm{~N} 1$, seasonal influenza virus, and critical illness in children. Lancet 2009; 374: 605-607.

4. ANZIC Influenza investigators. Critical care services and 2009 H1N1 Influenza in Australia and New Zealand. N Engl J Med 2009; 361: 1925-1934.

5. Jain S, Kamimoto L, Bramley AM, et al. Hospitalized patients with $2009 \mathrm{H} 1 \mathrm{~N} 1$ influenza in the United States, April-June 2009. N Engl J Med 2009; 361: 1935-1944.

6. Larcombe PJ, Moloney SE, Schmidt PA. Pandemic (H1N1) 2009: a clinical spectrum in the general population. Arch Dis Child 2009, [Epub ahead of print].

7. O'Riordan S, Barton M, Yau Y, Read SE, Allen U, Tran D. Risk factors and outcomes among children admitted to hospital with pandemic H1N1 influenza. CMAJ 2010; 182: 39-44.

8. Rothberg MB, Haessler SD. Complications of seasonal and pandemic influenza. Crit Care Med 2009, [Epub ahead of print].

9. Jain R, Goldman RD. Novel influenza A (H1N1): clinical presentation, diagnosis and management. Pediatr Emerg Care 2009; 25: 791-796.

10. Gupta S, Markham DW, Drazner MH, Mammen PP. Medscape. Fulminant myocarditis. Nat Clin Pract Cardiovasc Med 2008; 5 : 693-706. 